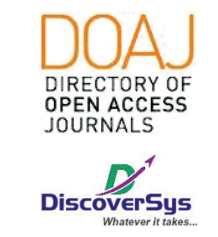

Published by DiscoverSys

\section{The influence of light physical activity on scale of pain in elderly with knee osteoarthritis}

\author{
Prasenah Subramaniam, ${ }^{1 *}$ Nyoman Astika, ${ }^{2}$ Ketut Mariadi ${ }^{2}$
}

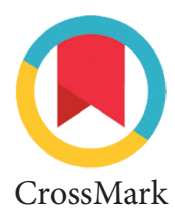

CrossMark

\title{
ABSTRACT
}

Background: Osteoarthritis $(0 \mathrm{~A})$ is a collection of mechanical variations of joint thinning, including the articular ligament and the subchondral bone. Aim: This study aims to find out how some light physical activity correlates with the pain scale among elderly with knee osteoarthritis and also to find out whether light physical activity in the elderly helps reduce pain in knee osteoarthritis.

Method: This research is a descriptive cross sectional study. The sample in this study was elderly people aged 50 years and above with a total of 70 samples were selected using multistage random sampling technique located in Badung Market, Kumbasari Market, and Renon Field. Data were obtained by interviewing respondents who previously provided informed consent using structured questionnaires. The degree of pain caused by osteoarthritis was measured using
The Western Ontario and McMaster University 0steoarthritis Index (WOMAC) and the total score.

Results: Distribution of sex within samples found that women (54.3\%) and men (45.7\%). Researchers found that the mean age of respondents was 61.2 (SD +3.75 ) years. The total number of respondents who have exercise habits is $52.9 \%$. The average WOMAC score was $23(S D+2,319)$ with a minimum score of 18 and a maximum of 27. Respondents with WOMAC score $<23$ were $52.9 \%$ whereas respondents with WOMAC score $>23$ were $47.1 \%$. Cross-tabulation results showed $97.3 \%$ of respondents with a WOMAC score $<23$ had exercise habits.

Conclusion: The exercise habits of the elderly with knee osteoarthritis can reduce pain when measured using a 0-4 scale and the WOMAC pain scale.
${ }^{1}$ Medical Science Study Program, Faculty of Medicine, Universitas Udayana

${ }^{2}$ Department of Internal Medicine, Faculty of Medicine, Universitas Udayana, Sanglah General Hospital Denpasar Bali
${ }^{*}$ Correspondence to:

Prasenah Subramaniam, Medical Science Study Program, Faculty of Medicine, Universitas Udayana prasenahsheena@yahoo.co.id

Received: 2018-06-28 Accepted: 2018-08-26 Published: 2019-08-01

Keywords: Sports, Osteoarthritis, Pain, WOMAC

Cite This Article: Subramaniam, P., Astika, N., Mariadi, K. 2019. The influence of light physical activity on scale of pain in elderly with knee osteoarthritis. Intisari Sains Medis 10(2): 215-219. D0I: 10.15562/ism.v10i2.293

\section{INTRODUCTION}

An osteoarthritis is a heterogeneous group of conditions that lead to signs and symptoms of joint. The disease is characterized by cartilage abrasion and their irregular new bone formation on the surface of the joints. Abnormalities of the cartilage will result in bones rub against each other, causing the symptoms of stiffness, pain, and limitation of motion in the joints. ${ }^{1}$ Risk factor for knee osteoarthritis include age over 50 years, female gender due to sex hormones, race/ethnicity, genetics, smoking, vitamin D consumption, obesity, osteoporosis, diabetes mellitus, hypertension, hyperuricemia, hysterectomy, meniscectomy, a history of knee trauma, anatomical abnormalities, the habit of working with heavy loads, heavy physical activity and exercise habits. ${ }^{2}$

Prone joints formed by the joint cartilage cells (chondrocytes) and cartilage matrix. Chondrocyte function to synthesize and maintain cartilage matrix of cartilage pads that function appropriately supported. Cartilage matrix is mainly composed of water, proteoglycans, and collagen. ${ }^{2}$ The development of osteoarthritis disease course is divided into three phases. Phase 1 is the occurrence of proteolytic decomposition in the cartilage matrix. Chondrocyte metabolism becomes affected and increases the production of enzymes such as metalloproteinases where later destroyed in the cartilage matrix. Chondrocytes also produce protease inhibitors that affect proteolysis. These conditions give manifestation to the depletion of cartilage. $^{2}$ Phase 2 where occurs fibrillation and erosion of the cartilage surface, accompanied by the release of proteoglycans and collagen fragments in the synovial fluid. The last is phase 3 where macrophage production of synovial such as interleukin 1 (IL-1), tumor necrosis factor-alpha (TNF- $\alpha$ ), and metalloproteinases be increased. These conditions give back to the cartilage manifestation and directly impact the destruction of cartilage. Pro-inflammatory molecules such as nitric oxide (NO) are also involved. These conditions provide the joint manifestation of architectural changes and impact on the growth of bone due to the stability of the joint. ${ }^{2}$

Osteoarthritis manifest as joint pain, rigidity, crepitus, swelling, the resistance of movement, reddening of the area and changes in gait. ${ }^{3}$ Diagnosis criteria for $\mathrm{OA}$, rely on clinical symptoms and radiologic finding. Diagnostic criteria based on American College of Rheumatology is showed in table 1 . One of the most concerning symptoms is 
joint pain. Joint pain usually increases because of movement and slightly reduced when a break. In certain movements (e.g., the knee is moved to the center) cause pain. The pain of osteoarthritis can spread another part, e.g., hip osteoarthritis causes calf pain known as "intermittent claudication." The correlation between pain and the degree of structural changes in osteoarthritis often found in hips, knees and rarely on hand and apofisis spinal joints. ${ }^{3}$ Pain is the typical symptoms in the joints experiencing osteoarthritis. More severe pain when doing activities with the use of joints and pain after activity caused by the use of joints and pain is getting lighter with a break. ${ }^{1}$ One way to overcome this pain is with physical therapy and light exercise. Physical therapy is used to train the patient so that the joints remain usable, and to instruct the patient to protect the joints. Physical therapy can make patients move as usual while reducing the physical risk that is not functioning correctly. Physical therapy in patients with osteoarthritis may include physiotherapy or light exercises such as cycling and swimming. Physical therapy is trying to do not give a too heavy burden on the patient. ${ }^{4}$ This study aims to know how does light physical activity correlates to pain scale of elderly with knee osteoarthritis and figure out whether light physical activity help reduces pain in elderly with knee osteoarthritis.

\section{METHOD}

This research is a descriptive study with a cross-sectional approach. The samples collected from Pasar Badung, Pasar Kumbasari and Lapangan Renon, Bali with multistage random sampling. The target population of this study is elderly above 50 years old. The variables that will be sorted in this research is age, gender, frequency and duration of exercise and the level of pain caused by knee osteoarthritis. Level of pain will be determined by using a scale of pain of 0-4. Each candidate will be interviewed on the level of pain experienced from knee osteoarthritis by using The Western Ontario and McMaster Universities Osteoarthritis Index (WOMAC) and the total score determines the pain caused by osteoarthritis, which means the higher the score, the more the pain. ${ }^{5}$ For exercise frequency, for example, simple stretching, aerobics, yoga and other light exercises data will be collected by considering at least once a week as frequent, at least once in 2 weeks as less frequent, at least once a month as rarely and never exercised as zero. The data for exercise duration is collected directly from how many hours sample exercise each time. ${ }^{10}$ Data will be collected through interview of elderly of a family of the elderly from markets around Badung, Pasar Badung and Pasar Kumbasari and Lapangan Renon. Data from the survey are primary data. Data thus collected will be analyzed, and relevant statistics will be calculated and presented through the graph and pie charts.

\section{RESULT}

The total of samples used in this study is 70 peoples. In the implementation of data collection, all respondents can fill out a questionnaire well. From 70 samples, it can be seen that the number of women as much as $38(54.3 \%)$ people more than the men who are 32 people (45.7\%). The average age of respondents was $61.2(\mathrm{SD}+3.75)$ years old with the youngest $53 \mathrm{y} / \mathrm{o}$ and the oldest $72 \mathrm{y} / \mathrm{o}$. Age was grouped based on Kolgomorov-Smirnov testing; it's found that age was normally distributed.

Respondents were mostly in the age group $<61.2$ years old (57.1\%). Table 3 shows the exercise profile of the respondents. It was found that more than half of respondents had a habit to exercise (52.9\%). Most of the respondents who regularly exercise (16 respondents) claimed to apply more than once a week. Most of the samples did training for 1 hour per session (73.3\%).

WOMAC index scores grouped based on the Kolmogorov-Smirnov test and it was found that the data were normally distributed. Therefore, the authors use the average of the WOMAC Score as

Table 1 Diagnostic criteria according to ACR CLINICAL KNEE JOINTS

\section{RADIOGRAPHIC}

Knee pain+ minimum (3) of the 6following criteria:

Knee pain + minimal one from the following criteria

a) Age $>50$ years

b) Rigid morning $<30 \mathrm{~min}$

c) crepitus changes

a) Osteophyte

d) Tenderness

e) Enlargement of bone

f) No heat on palpability

b) Narrowing of the gap joints often asymmetric of the joint anatomical structure.

c) Subchondral cysts and sclerosis 
Table 2 Characteristics of sample

\begin{tabular}{llcc}
\hline Variables & & Frequency $\mathbf{( N = 7 0 )}$ & Percentage (\%) \\
\hline Gender & Male & 32 & 45.7 \\
& Female & 38 & 54.3 \\
\multirow{2}{*}{ Age Group } & $<61.2$ y/o & 40 & 57.1 \\
& $>61.2$ y/o & 30 & 42.9 \\
\hline
\end{tabular}

Table 3 Sample's exercise habit

\begin{tabular}{llcc}
\hline Variables & & Frequency & Percentage (\%) \\
\hline Exercise Habit & Exercise & 37 & 52.9 \\
Type of Exercise $(\mathrm{N}=37)$ & No Exercise & 33 & 47.1 \\
& Aerobics & 4 & 10.8 \\
& Light Stretching & 14 & 37.8 \\
& Walking & 9 & 24.3 \\
Exercise Frequency $(\mathrm{N}=37)$ & Yoga & 10 & 27.1 \\
& $>$ Once in a week & 18 & 48.6 \\
& Once in two weeks & 14 & 37.8 \\
Duration of Exercise $(\mathrm{N}=37)$ & Once a month & 6 & 13.6 \\
& $<30$ minutes & 7 & 18.9 \\
& $<1$ hour & 2 & 5.4 \\
& 1 hour & 26 & 73.3 \\
& $>1$ hour & 2 & 5.4
\end{tabular}

Table 4 Cross tabulation between a group of WOMAC score and other variables

\begin{tabular}{|c|c|c|c|c|c|}
\hline & & \multicolumn{4}{|c|}{ WOMAC Score Index } \\
\hline & & \multicolumn{2}{|c|}{$<23(\mathrm{~N}=37)$} & \multicolumn{2}{|c|}{$>23(\mathrm{~N}=33)$} \\
\hline & & Frequency & Percentage & Frequency & Percentage \\
\hline \multirow[t]{2}{*}{ Gender } & Male & 18 & $48.6 \%$ & 14 & $42.4 \%$ \\
\hline & Female & 19 & $51.4 \%$ & 19 & $57.6 \%$ \\
\hline \multirow[t]{2}{*}{ Age } & $<61.2 \mathrm{y} / \mathrm{o}$ & 33 & $89.1 \%$ & 7 & $21.2 \%$ \\
\hline & $>61.2 \mathrm{y} / \mathrm{o}$ & 4 & $10.9 \%$ & 26 & $78.8 \%$ \\
\hline \multirow[t]{2}{*}{ Exercise Habit } & Yes & 36 & $97.3 \%$ & 1 & $3 \%$ \\
\hline & No & 1 & $2.7 \%$ & 32 & $97 \%$ \\
\hline \multirow[t]{4}{*}{ Type of Exercise } & Aerobics & 4 & $10.8 \%$ & 0 & $0 \%$ \\
\hline & Light Stretching & 13 & $35.1 \%$ & 1 & $3 \%$ \\
\hline & Walking & 9 & $24.3 \%$ & 0 & $0 \%$ \\
\hline & Yoga & 10 & $29.9 \%$ & 0 & $0 \%$ \\
\hline \multirow[t]{3}{*}{ Frequency of Exercise } & $>$ Once in a week & 16 & $43.2 \%$ & 0 & $0 \%$ \\
\hline & Once in 2 weeks & 16 & $43.2 \%$ & 1 & $3 \%$ \\
\hline & Once in a Month & 4 & $13.6 \%$ & 0 & $0 \%$ \\
\hline \multirow[t]{4}{*}{ Duration of Exercise } & $<30$ minutes & 6 & $16.2 \%$ & 1 & $3 \%$ \\
\hline & $<1$ hour & 2 & $5.4 \%$ & 0 & $0 \%$ \\
\hline & 1 hour & 26 & $73 \%$ & 0 & $0 \%$ \\
\hline & $>1$ hour & 2 & $5.4 \%$ & 0 & $0 \%$ \\
\hline
\end{tabular}


a cut of point. The average WOMAC score is 23 with standard deviation 2,319 and minimum value 18 and maximum 27. Respondents with WOMAC score $<23$ were 37 people (52.9\%) while respondents with WOMAC score $>23$ were 33 persons (47.1\%).

Table 4 illustrates the frequency distribution and percentage of WOMAC scores based on other variables. Based on the respondent's gender, a group with a WOMAC score $<23,51.4 \%$ is female, and the rest are male. Whereas if the WOMAC score is seen based on the age of the respondents, then the respondents who have a WOMAC score $<23$ $89.1 \%$ are $<61.2$ years old. When we look at the habit patterns of exercise, then $97.3 \%$ of respondents with a WOMAC score $<23$ have exercise habits. Most respondents (35.1\%) with a WOMAC score $<23$ chose light stretching as their preferred exercise type. A total of $43.2 \%$ of respondents with a WOMAC score $<23$ had exercise habits more than twice a week and at least once in 2 weeks. And as many as $73 \%$ of respondents with a WOMAC score $<23$ did exercise at least 1 hour in each session.

\section{DISCUSSION}

\section{WOMAC Score Based On Gender}

Based on sex differences, groups with lower WOMAC scores are predominantly female. The women tend to have higher WOMAC scores than men $(\mathrm{p}<0.001)$ because women's pain thresholds are lower than men. ${ }^{5}$ Studies from Paradowski show that there is no relationship between sex and the scale of perceived pain. ${ }^{6}$ Study from Kulcu et al. supported this research finding, it stated that WOMAC Score is higher among women $(\mathrm{p}=0.024)$, but they didn't explain the reason behind it. ${ }^{7} \mathrm{~A}$ lower WOMAC score in women in this study may be due to the majority of the sample being female.

\section{WOMAC Score Based On Age}

Based on the age group, most respondents with lower WOMAC scores were from the younger age group. The results of this study are similar to those of Singh et.al. Based on a study of Singh et al. who reviewed WOMAC scores in a population aged 45-85 years, it was found that the WOMAC score increased with age of patients with the highest score belong to the group of 80 -year age group $(r=0.782$; $\mathrm{p}=0.0001) .{ }^{1}$ In contrast, a study from Kulcu et al. showed that there was no significant difference between age group and WOMAC score $(\mathrm{p}=0.09){ }^{7}$

\section{WOMAC Score Based On Exercise Habit}

Most respondents with lower WOMAC scores came from groups who had exercise habits. Research from Kulcu et al. showed that physical activity has a significant relationship to WOMAC Score, where patients with more physical activity tend to have lower WOMAC scores $(r=0.352 ; p=0.003)$. This is associated with a decrease in BMI in subjects who actively perform activities compared with those who never perform physical activity. Lower BMI was also shown to be associated with a lower WOMAC score $(\mathrm{r}=0.749 ; \mathrm{p}=0.0001){ }^{7}$ The study of Avelar et al. also supports the fact that lower BMI correlates with lower WOMAC score $(r=0.364 ; p=$ $0.001) .{ }^{8}$ Given the results of this kind of research, it can be concluded that exercise activities will cause BMI to decrease and cause low WOMAC score on respondents. The results of Rhon et al. also showed that exercise programs performed in patients with osteoarthritis for 1 month resulted in improved pain indicated by a decrease in WOMAC score $(\mathrm{p}=0.001)$ with an initial mean of WOMAC score of 105 to 56 within 1 month. Patients also did not experience exacerbations of pain within 72 hours after undergoing exercise sessions. ${ }^{9}$

\section{WOMAC Score Based On Type of Exercise}

Most respondents with lower WOMAC scores were from groups who had a light stretching exercise habit. This result is in contrast to the Kirihara et al. study, where the results show that the more severe the physical activity, the more significant the WOMAC score decreases. In the study, the sample performed aerobic, yoga, walking, weight lifting, stretching, tai chi chian, biking, swimming and pilates exercises. And the activity that significantly decreased the WOMAC score was weightlifting ( $p$ $=0.009)$ and swimming $(\mathrm{p}=0.003)$. It is also associated with the effectiveness of physical activity in decreasing BMI to reduce the WOMAC score. ${ }^{10}$ In this study found that in the group with lower WOMAC score more that do the movement of light stretching because indeed most of the samples do physical activity in the form of light stretching. This is reasonable given the population used in this study is the elderly population, whereas the population used in the Kirihara et al. study tends to be younger, so it is more natural to perform more severe activities.

\section{WOMAC Score Based on Exercise Frequency} Based on the frequency of exercise, most respondents with lower WOMAC scores are from groups who have exercise habits more than once a week. This is consistent with the results of the study from Kirihara et al., Where patients who engage in any physical activity, tend to have lower WOMAC score in contrast with sedentary patients $(\mathrm{p}=0.02) .{ }^{10}$

\section{WOMAC Score Based on Exercise Duration}

Based on the duration of exercise, most respondents with lower WOMAC scores were from the group 
who had exercise habits with span of at least 1 hour in a session. Research from Kirihara et al. also showed that patients who exercised at least 1 hour each session experienced decreased pain in their joints so after 2 years of undergoing exercise program the patient could exercise up to 180 minutes per session ( $\mathrm{p}<0.001) .{ }^{10} \mathrm{~A}$ meta-analysis study from Regnaux et al. showed that there was a statistically significant $(-0.84 ; 95 \% \mathrm{CI}-1.63$ to $-0.04 ; \mathrm{p}=0.04)$ decline of 20 points on the WOMAC pain scale in the sample performing the exercise program with higher intensity compared to the low intensity. Decreased absolute pain in $4 \%$ of the sample and relative pain relief in $13 \%$ of the sample. In this study, high-intensity exercise was performed at least one hour per session with a total of courses of at least twice a week. ${ }^{11}$ Intensity and frequency of exercise can affect WOMAC score in correlation with decreasing BMI too. ${ }^{7}$

\section{Limitation}

The participants or sample may provide false information and may not answer the details explicitly required due to communication problems and their understanding of the research because of age. The explanation must be given right during the distribution of questioners

\section{CONCLUSION}

Physical activity among osteoarthritis patients can reduce the pain reflected in the decrease of the WOMAC score index. The more engaged the patients in the exercise program, the more decrease the WOMAC score index. The more duration of exercise session also can be associated with lower WOMAC index score. In this study, we found that a lighter type of exercise has more samples in the lower WOMAC group. Women gender and older age have more people in higher WOMAC Group.
Further research needed to analyze correlation within exercise and pain intensity in osteoarthritis patients. We also need to determine the cutoff point of the type, frequency, and severity of the exercise that wouldn't harm the patients.

\section{REFERENCES}

1. Singh Sudhir, Khanna Vikram. Correlation between clinical and radiological grading of osteoarthritis. Science J Med Sci. 2014; 3(5): 320-324

2. Behzad Heidari. Knee osteoarthritis prevalence, risk factors, pathogenesis, and features. Caspian J Intern Med. 2011; 2(2): 205-212.

3. Sharma Leena. Osteoarthritis Year in Review 2015: Clinical. Osteoarthritis Cartilage. 2016; 24(1): 36-48

4. Esser S, Bailey A. Effects of exercise and physical activity on knee osteoarthritis. Curr Pain Headache Rep; 15: 423-430

5. Fang WH, Huang GS, Chang HF, et al. Gender differences between WOMAC index scores, health-related quality of life and physical performance in elderly. BMJ Open. 2015; 5(9): e008542

6. Paradowski Przemyslaw, Englund Martin, Lohmaner Stefan, et.al. The effect of patient characteristics on variability in pain and function over two years in early knee osteoarthritis. Health Qual Life Outcomes. 2015; (3):59-63

7. Kulcu DG, Yanik Burcu, Atalar Hakan, et.al. Associated Factors with Pain and Disability in Patients with Knee Osteoarthritis. Turk J Rheumatol. 2010; 25: 77-81

8. Avelar ML, Gomes WF, Zille B, et.al. Muscle Performance, pain, Stiffness, and functionality in elderly women with knee osteoarthritis. Acta Ortop Bras. 2011; 19(4)

9. Rhon D, Deyle G, Gill N, et.al. Manual physical therapy and perturbation exercise in knee osteoarthritis. J Man Manip Ther. 2013; 21(4):220-228

10. Kirihara AR, Catelan FB, Sabino FE, et.al. Intensity, Duration, and Type of Physical Activity Required to Improve Function in Knee Osteoarthritis. Acta Ortop Bras. 2017.; 25(1): 25-29

11. Regnaux Jp, Volau Lefevre, Trinquat L, et.al. High Intensity versus low-intensity physical activity or exercise in people with hip or knee osteoarthritis. The Cochrane Collaboration; 2015.

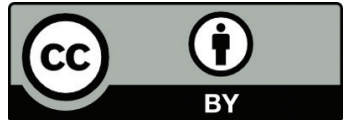

This work is licensed under a Creative Commons Attribution 